\title{
PREVALENCE OF MICROALBUMINURIA AMONG TYPE 2 DIABETIC PATIENTS IN ILORIN, NIGERIA
}

\author{
AbdulRahman MB ${ }^{1}$, Okesina AB ${ }^{2}$, Makusidi MA ${ }^{3}$, Muftau YA ${ }^{4}$, Shabbal DA $^{5}$ \\ ${ }^{1}$ Department of Chemical Pathology and Immunology, College of Health Sciences, Usmanu Dafodiyo University, Sokoto, \\ ${ }^{2}$ Department of Chemical Pathology and Immunology, College of Health Sciences, University of Ilorin, Kwara State. \\ ${ }^{3}$ Department of Medicine, College of Health Sciences, Usmanu Dafodiyo University, Sokoto. \\ ${ }^{4}$ Department of Psychiatry, College of Health Sciences, Usmanu Dafodiyo University, Sokoto. \\ ${ }^{5}$ Department of Chemical Pathology, University of Abuja Teaching Hospital, Gwagwalada, Abuja \\ Correspondence: MB AbdulRahman \\ Department of Chemical Pathology and Immunology, College of Health Sciences, Usmanu Dafodiyo University, Sokoto. \\ email: abfmhz@yahoo.com
}

\section{ABSTRACT}

Background: Microalbuminuria is predictive of renal failure in diabetes and is also an independent predictor of cardiovascular and all causemortalityboth in diabetes and in general population. The three leading causes of chronic kidney disease (CKD) in Nigeria are chronic glomerulonephritis, hypertension and diabetes mellitus, while diabetes mellitus is the leading cause of CKD in developed countries.

Objective: The aim of this study was to determine the prevalence of microalbuminuria among type 2 diabetic patients attending diabetic clinic of University of Ilorin Teaching Hospital, Ilorin Kwara state, Nigeria.

Methods: One hundred and thirty two known type 2 diabetic patients and sixty six controls were recruited for this study.Urinary albumin concentration was determined using ELISA method, data was collated and analysed using SPPSS version 20.

Results:The overall prevalence of microalbuminuria in this study was $37.9 \%$. The prevalence of microalbuminuria increased with poor glycaemic control and duration of diabetes.Multivariate regression analysis revealedthat,the age,glycated haemoglobin,fasting plasma glucose and duration of diabetes mellitusare associated with microalbuminuria.

Conclusion: The prevalence of microalbuminuria in type 2 diabetic patients in this cross-sectional study was $37.9 \%$ and this was significantly related to age andpoor metabolic control.

Keywords: Prevalence, microalbuminuria, type 2 diabetics, Ilorin

\section{INTRODUCTION}

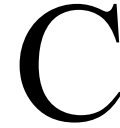

hronic kidney disease (CKD) in Nigeria mainly affects young adults in their most productive years there by constituting a drain on the nations economy ${ }^{1,2}$. The three leading causes of CKD in Nigeria are chronic glomerulonephritis, hypertension and diabetes mellitus $^{3,4}$ while diabetes mellitus is the leading cause of CKD in developed countries 5 .

Microalbuminuria is predictive of renal failure in diabetes and is also an independent predictor of cardiovascular and all causemortality both in diabetics and in general population ${ }^{6,7}$. The presence ofmicroalbuminuria, glomerular hyperfiltration and hypertension are markers of renal disease in diabetes and therefore their presence predict the development of clinical diabetic nephropathy ${ }^{6,7}$.

It is established that early detection and proper management of diabetic nephropathy are imperative in achieving regression of CKD and retardation of its progression to end stage renal disease. This study therefore aimed at determining the prevalence of microalbuminuria among type 2 diabetic patients as seen in University of Ilorin Teaching Hospital, Ilorin, North Central geopolitical zone of Nigeria.

\section{MATERIALS AND METHOD}

This was a cross sectional descriptive study among type 2 diabetes patients in the North central geopolitical zone of Nigeria. Type 2 diabetes mellitus was diagnosed using the World Health Organization (WHO) criteria ${ }^{8}$. Patients with history of hypertension and/or renal disease were excluded. One hundred and thirty two type $2 \mathrm{DM}$ patients who met above criteria and consented to the study were recruited. Theywere age and sex matched with 66 healthy subjects who served as control in a ratio of $2: 1$. The anthropometric measurement was obtained and $5 \mathrm{mls}$ of venous blood in fasting statewas obtained after observing aseptic procedure. The blood was collected into a lithium heparin bottle for analysis. Spot urine sample was collected into a clean plain specimen bottle for d e t e r m in a t i o n of microalbuminuria by immunoturbidometric assay.

The data was analysed using 
SPSS version 16 and were presented as mean, standard deviation (SD) and percentages. Student's t-testwas used to compare means and chi squared test to compare proportions. $\mathrm{P}$ values $<0.05$ were considered significant.

Biochemical analyses were done on Ciba Corning Express Plus Auto Analyser (Corning, Medfield, MA, USA) using kits supplied by Boehringer Mannheim, (Mannheim, Germany). Fasting and postprandial plasma glucose was estimated byglucose oxidase method. Glycated haemoglobin (HbA1c) was estimated by high pressure liquid chromatography using the Variant machine (Bio Rad, Hercules, CA, USA).

Spot urine samples were collected andurine creatinine was measured using Kinetic method. Urine microalbumin concentration was measured using commercially available immnuoturbidometric assay kits from Randox (Randox, UK) on Opera Technicon Auto Analyser (Bayer Diagnostics, USA). The urine sample was added to a buffer containing antibody specific for human serum albumin. The absorbance of the resulting turbid solution is proportional to concentration of albumin in the sample solution. By constructing a standard curve from the absorbances of the standards, the albumin concentration in the sample can be determined. The mean interassay and intraassay coefficient of variation were $3.4 \%$ and $2.4 \%$ respectively.

\section{RESULTS}

The overall prevalence of microalbuminuria in this study was $37.9 \%$. The mean age \pm SD of the patients was 59.53years \pm 0.86 years, mean $\mathrm{BMI} \pm \mathrm{SD}$ was $31.81 \pm 0.54 \mathrm{~kg} / \mathrm{m}^{2}$ and the mean age \pm SD was $58.9 \pm 1.63$ years.
Ninety three $(70.5 \%)$ patients were females with mean age of $59.9 \pm 1.01$ years and thirty nine $(29.5 \%)$ were males.

Systolic Blood Pressure (SBP), Diastolic Blood Pressure (DBP), fasting Plasma Glucose (FPG), glycated haemoglobin (HBAIC), albumin creatinine ratio (ACR) and estimated glomerular filtration ration (eGFR) were $126.70 \pm 1.08,87.56 \pm .97$, $8.52 \pm 0.49,9.19 \pm 0.47,11.79 \pm$ 1.07 and $62.83 \pm 6.77$ respectively. Univariate analysis demonstrated significant a s s o c i a tion betwe e $n$ microalbuminuria and poor glycaemic control, duration of diabetic and female gender.

\section{DISCUSSION}

The incidence of DM especially type 2 is rapidly growing worldwide with some of its attendant chronic complications such as diabetic nephropathy (DN). Albuminuria is a renal risk factor and a sign of endothelial dysfunction which may lead to overt renal impairment. In this present study microalbuminuria occurred in $37.9 \%$ of type 2 diabetic patients which is comparableto the prevalencerate of $38 \%$ reported by Orluwene et $\mathrm{al}^{(9)}$ in Port Harcourt and $37.6 \%$ in Lagos by Iwalokun et $\mathrm{al}^{(10)}$. Our findings concur with the overall global prevalence of microalbuminuria of $39 \%$ in the study carried out by the Development of Education on microalbuminuria for Awareness of Renal and Cardiovascular Risk Diabetes Study. The prevalence in our study is however, higher than the prevalence of $25 \%$ reported by Adebisi et $\mathrm{al}^{(11)}$ in Ilorin, $21 \%$ reported by Khihava et al ${ }^{(12)}, 20 \%$ by Torffvitt et al ${ }^{(13)}$ and $22 \%$ as reported by Parvin et $\mathrm{al}^{(14)}$ all among type 2 diabetic patients. However, our finding contrast with the prevalence of $52 \%$ reported by Erasmus et $\mathrm{al}^{(15)}$, in a study conducted in Ilorin, 50\% reported in Benin by Unuigbe et $\mathrm{al}^{(16)}$ and $42 \%$ prevalence was reported by Okesina et $\mathrm{al}^{(17)}$ in South Africa. The variation in most of thestudies quoted above could be due to patient sample size, various assay methodology of albuminuria, and probably early commencement of drugs to reduce the risk factors. Some of the reasons for the higher prevalence rates have been linked to hyperglycaemia (poor glycaemic control) and obesity, these are independent strong risk factors for developing proteinuria. We observed anincrease in the mean of body mass index, fasting plasma glucose and glycated haemoglobin for the diabetic patients. However, the variation in the prevalence rate is probably attributable to difference in diagnostic criteria, the stage of the disease and the method of assessment.

Furthermore, no significant correlation was observed between the degree of glycaemic control and albuminuria. These findings are similar to observations made amongst diabetics in Nigeria by Erasmu et al ${ }^{(18)}$ and in Mexico by Hafnner et al ${ }^{(19)}$ respectively. An interesting observation in this study was the high prevalence of microalbuminuria in patients with relatively short duration of DM less than 5 years. This is similar to findings of Erasmu et al ${ }^{(20)}$.

Micro-albuminuria occurred at all known duration of diabetes and the data in this study supports that abnormal albumin excretion may be detectable shortly after diagnosis. Similar findings have been reported from Nigeria by Erasmus et al ${ }^{(18)}$, from Nauru by Collins et al and from Saudi Arabia by Alzaid et al ${ }^{(21)}$ but are in contrast to other studies in which duration of diabetes was found to be a strong predictor of microalbuminuria as reported by 
Table 1 Characteristics of type 2 diabetic patients $($ mean \pm SD)

\begin{tabular}{lrccc}
\hline Parameters & Patient & Control & t test & P - value \\
\hline Number & $\mathbf{1 3 2}$ & $\mathbf{6 6}$ & & \\
Age & $59.5 \pm 0.86$ & $62.4 \pm 1.32$ & 17.35 & $<0.0001$ \\
BMI & $31.8 \pm 0.54$ & $26.81 \pm 0.84$ & 4.867 & $<0.0001$ \\
FPG & $8.03 \pm 0.29$ & $4.12 \pm 0.21$ & 7.829 & $<0.0001$ \\
HBA1C & $8.84 \pm 0.27$ & $3.40 \pm 0.15$ & 12.04 & $<0.0001$ \\
SBP & $127.91 \pm 0.72$ & $118.80 \pm 0.74$ & 6.936 & $<0.0001$ \\
DBP & $87.61 \pm 0.58$ & $80.70 \pm 0.90$ & 6.707 & $<0.0001$ \\
\hline
\end{tabular}

Table 2: Clinical and biochemical characteristic of the study subjects

\begin{tabular}{lllll}
\hline & Male & Female & t-test & P-value \\
\hline Number & $39(29.5 \%)$ & $93(70.5 \%)$ & & \\
Age (years) & $58.9 \pm 1.63$ & $59.9 \pm 1.01$ & 3.556 & 0.0008 \\
Duration (year) & $3.77 \pm 0.697$ & $4.66 \pm 0.433$ & 7.398 & $<0.0001$ \\
BMI & $29.49 \pm 0.991$ & $32.67 \pm 0.616$ & 22.363 & $<0.0001$ \\
FPG $(\mathrm{mmol} / \mathrm{L})$ & $8.53 \pm 0.644$ & $7.71 \pm 0.310$ & 9.881 & $<0.0001$ \\
$\mathrm{HBA}_{1} \mathrm{c}$ & $9.69 \pm 0.52$ & $8.50 \pm 0.315$ & 16.146 & $<0.0001$ \\
$\mathrm{SBP}(\mathrm{mmHg})$ & $127.85 \pm 1.65$ & $127.72 \pm 0.79$ & 0.613 & 0.5412 \\
$\mathrm{DBP}(\mathrm{mmHg})$ & $85.56 \pm 0.99$ & $87.85 \pm 0.67$ & 15.443 & $<0.0001$ \\
\hline
\end{tabular}

TABLE 3 : Prevalence of microalbuminuria and macroalbuminuria in relation to duration of diabetes

\begin{tabular}{llcc}
\hline $\begin{array}{l}\text { Duration of } \\
\mathrm{DM}(\mathrm{yrs})\end{array}$ & $\mathrm{N}(\%)$ & microalbuminuria & macroalbuminuria \\
\hline $0-5$ & $103(78.3 \%)$ & $40(38.8 \%)$ & $8(7.8 \%)$ \\
$6-10$ & $2(15.9 \%)$ & $7(33.3 \%)$ & $1(4.8 \%)$ \\
$>11$ & $8(6.06 \%)$ & $3(3 \%)$ & $0(0 \%)$
\end{tabular}

diabetics to present with e $\mathrm{s} t \mathrm{ablis}$ e d complications.

As reported in some studies such as Parvin et al ${ }^{(24)}$, Erasmus et al ${ }^{(18)}$, and Mclarty et al $(1992)^{(23)}$, African d i a b e t i c s w ith hypertension and e levated s e rum creatinine were at greater risk of developing micro-albuminuria. This study, however, showed similar blood pressure levels in normo, micro and macro-albuminuric diabetics. These findings are in contrast to studies which have emphasized the importance of the relationship between UAE and elevated blood pressure Erasmus et al ${ }^{(18)}$, Alzaid et al ${ }^{(2)}$. This may probably be due to antihypertensive administration by some patient.

\section{CONCLUSION}

The overall prevalence of microalbuminuria in this study was $37.9 \%$. DN was more prevalent amongst the newly diagnosed patients with type 2 diabetes. There is an association between microalbuminuria and poor glycaemic control, duration of diabetes and female gender.

We recommend that preventive measures such as stepping up glycaemic control and early antihypertensive

West et al ${ }^{(22)}$, Mc Larty et al ${ }^{(23)}$,Albeert et al and Iwalokun et al at Lagos.

The se conflicting observationsmay have occurred due to difficulty in dating the onset of type 2 diabetes which may be connected with low education levels in some patients. In many developing countries like Nigeria, DM is undetected for a long period of time and is not uncommon for newly diagnosed treatment with ACE inhibitors need to be intensified. Furthermore, regular screening for microalbuminuria should be implemented as part of the routine care of diabetic patients in Nigeria. 


\section{References:}

1. Akinsola W, Odesanmi WO, Oguniyi JO, Ladipo GO. Diseases causing chronic renal failure in Nigeriansprospective study of 100 cases. Africa J. Med.\&Med Sci. 1989; 18:131-137

2. Ojogwu LI. The pathological basis of end stage renal disease in Nigerians: experience from Benin City. West Afr J Med1990; 9:193196

3. Alebiosu CO, Ayodele OO, Abbas A, Olutoyin Al. Chronic renal failure at the Olabisi Onabanjo University Teaching Hospital, Sagamu, Nigeria. Afr Health Sci. 2006; 6:132-138

4. Ulasi I, Ijoma CK. The enormity of chronic kidney disease in Nigeria: The situation in a teaching hospital in south-east Nigeria. J.Trop Med.2010;1-6

5. United State Renal Data System. Excerpts from the united renal data system 2001data report: Atlas of end stage renal disease in the United States. Am. J. Kidney Dis. 2001; 38:1-248

6. Macleod J, Lutale J, Marshall S. Albumin excretion and vascular deaths in NIDDM Diabetologia 1995; 38:610616

7. Dinnen S, Gerstein H. The a s s o c i a t i o n of Microalbuminuria and mortality in NIDDM. A systematic over view of the literature. Arch Int Med 1998; 1413-1418

8. World Health Organization. Definition, diagnosis and classification of diabetes mellitu and its complications. WHO, Geneva. 1998.

9. Orlumene CG Mohom MO: $\mathrm{S} c \mathrm{r}$ e e $\mathrm{ning}$ for Microabuminuria in newly diagnosis type II at staff clinic in Port-Harcourt. Med J.
2008:10-14.

10. Iwalokun BA, Ounfemi MK, Gbajabiamila B, Olukosi YA. Incidence and evaluation of $\mathrm{r}$ i s k f a c t o r of Microalbuminuria among diabetics and non-diabetics in Lagos, Nigeria. Nig.J Health and Biomedical Sci. 2006;3:80-82

11. Adebisi SA, Okesina AB, Abu EO. Microalbuminuria in type II diabetic patients in Ilorin: A study of prevalence, sex distribution and relationship to blood pressure, metabolic control and body mass index. Sahel Med J, 2002; 5:39-42.

12. Kikkawa R, Umemura., Haneda M, Arimura T., Ebata K, Shigeta Y. et al, Evidence of polyol pathway in cultural rate mesangial cells. Diabates 1987; 36:240-247

13. Torffvit O, Agardh E, Agardh $\mathrm{E}$, Agardth CD: Albuminuria and associated medical risk factors: a cross sectional study in 451 type 2 diabetic patients. J. Diabet Compl. 1991; 5: 29-34

14. Parvin HH, Hommel E, Mathiesen E, Skott P, Edsberg $B$, Bahnsen $M$. et al. $\mathrm{P}$ e vale $\mathrm{nc}$ e of Microalbuminuria, arterial hypertension, retinopathy and neuropathy in patients with insulin dependent diabetes. Br Med J (Clin Res Ed). 1988; 296: 156-160.

15. Erasmus R T, Oyeyinka G O, Arije A. Microalbuminuria in non-insulin-dependent (type II) Nigerian diabetics: relation toglycemic control, blood pressure and retinopathy. Post grad Med J 1992; 68:638-642.

16. Umuigbe E.I, Omeifa $\mathrm{H}$, Edema T, Ukoli FA. Microalbuminuria and associated factors in newly diagnosed diabetes. Niger Postgrad Med J. 2001; 8: 187 192

17. Erasmus RT, Okesina B:
Microalbuminuria in a clinic population of type II Melanesian diabetics: Relationship to glycaemic control, blood pressure and duration of diabetes.Cent. Aft J. Med 1999; 45:291-294

18. Erasmus RT, Oyeyinka G O, Arije A. Microalbuminuria in non-insulin-dependent (type II) Nigerian diabetics: relation to glycaemic control, blood pressure and retinopathy. Post grad Med J 1992; 68:638-642.

19. Haffner SM, Stern MP Mitchell BD increase $\mathrm{p} \mathrm{r} \mathrm{e} \mathrm{vale} \mathrm{n} \mathrm{c}$ e of microalbuminuria in Mexican Americans, a high risk population for non-insulin dependent diabetes mellitus. Proceed of the 31 th International Diabetes Federation Congress.1988, Hobat Tasmania.

20. Alzaid AA,Sobki S,De Sliva V. Prevalence of microalbuminuria in Saudi Arabians with non-insulin dependent diabetes mellitus: a clinic based study. Diab Res \& Clin Pract, 1994; 26: 115120

21. West KM, Erdrerch LJ, Strober JA. A detailed study of factors for retinopathy and nephropathy in diabetes. Diabetes 1980; 501-508

22. Mclarty D.G Pollitt C. Swai Abm: Diabetes in African part 2. Pract. Biabetes 1992; 3 (2) 35-40

23. $\mathrm{P}$ a $\mathrm{r}$ i n g H.H Microalbuminuria in Essential Hypertension and Diabetes mellitus. J. of Hypertension 1996; 14: 569575

.24.Marshall, SM \& Alberti KG M.M Comparison of the prevalence association features of abnormal albumin excretion in insulin dependent and non-insulin dependent diabetes. QJ Med 1989: 61-71 